Die Schweiz und die humanitäre Aktion

\title{
Entwicklung der Humanitären Hilfe des Bundes und der schweizerischen Hilfswerke (1990-1997)
}

Gérard Perroulaz

\section{OpenEdition}

Journals

Electronic version

URL: http://journals.openedition.org/sjep/626

DOI: 10.4000/sjep.626

ISSN: 1663-9677

Publisher

Institut de hautes études internationales et du développement

Printed version

Date of publication: 1 janvier 1999

Number of pages: 9-23

ISSN: 1660-5926

\section{Electronic reference}

Gérard Perroulaz, « Entwicklung der Humanitären Hilfe des Bundes und der schweizerischen

Hilfswerke (1990-1997) », Schweizerisches Jahrbuch für Entwicklungspolitik [Online], 18| 1999, Online erschienen am: 18 Juli 2012, abgerufen am 08 September 2020. URL : http://journals.openedition.org/ sjep/626 ; DOI : https://doi.org/10.4000/sjep.626 


\title{
ENTWICKLUNG DER HUMANITÄREN HILFE DES BUNDES UND DER SCHWEIZERISCHEN HILFSWERKE (1990-1997)
}

\author{
Gérard Perroulaz
}

\section{ENTWICKLUNG DER HUMANITÄREN HILFE DER DAC-LÄNDER'}

Die OECD gibt einige globale Schätzungen zur humanitären Hilfe heraus. Die Nothilfe hatte seit 1990 stark zugenommen und hatte 1994 einen Höhepunkt erreicht. Das DAC hatte 1994 den Gesamtbetrag der humanitären Hilfe der Mitgliedsländer (einschliesslich Flüchtlingshilfe) auf mehr als 3,5 Milliarden Dollar geschätzt. Die über die UNICEF und das UNHCR, das Welternährungsprogramm und die Europäische Union laufende multilaterale humanitären Hilfe betrug ihrerseits rund 3 Milliarden Dollar. Die (nicht in der öffentlichen Entwicklungshilfe eingeschlossenen) militärischen Aufwendungen zur Friedenssicherung sind in den letzten Jahren ebenfalls erheblich gestiegen. Diese Friedenssicherungseinsätze beliefen sich 1994 auf rund 3,5 Milliarden Dollar, davon fast die Hälfte allein für das Gebiet Ex-Jugoslawien² 1996 erreichte die bilaterale humanitären Hilfe 3 Milliarden Dollar und die multilaterale humanitären Hilfe 2,5 Milliarden Dollar, ohne die 3 Milliarden Dollar für die (nicht unter der öffentlichen Entwicklungshilfe verbuchten) Kosten der Friedenssicherungseinsätze zu berücksichtigen. Nach obigen Schätzungen ist die gesamte humanitäre Hilfe zwischen 1994 und 1996 von 10 auf 8,5 Milliarden Dollar zurückgegangen.

\section{ENTWICKLUNG DER HUMANITÄREN HILFE DES BUNDES}

Der Auftrag der Eidgenossenschaft ist im Bundesgesetz von 1976 über die internationale Entwicklungszusammenarbeit und humanitäre Hilfe umschrieben: «Die humanitäre Hilfe soll mit Vorbeugungs- und Nothilfemassnahmen zur Erhaltung gefährdeten menschlichen Lebens sowie zur Linderung von Leiden beitragen; sie ist namentlich für die von Naturkatastrophen oder bewaffneten Konflikten heimgesuchte Bevölkerung bestimmt. » Die Jahresübersicht im vorliegenden Jahrbuch liefert Informationen über Organisation und Einsatzmittel im Rahmen dieses Instruments der internationalen Zusammenarbeit, mit den neuesten Daten über die humanitäre Hilfe des Bundes.

Das folgende Schema fasst die Einsatzformen der humanitären Hilfe zusammen. Der Bund leistet zwei Arten von Hilfe: direkte Einsätze, vor allem durch das Schweizerische Katastrophenhilfekorps (SKH, rund 15\% der Leistungen), und Unterstützung von Partnerorganisationen (multilaterale Organisationen, IKRK und schweizerische Hilfswerke). Der Bund verfügt über vier

* Forschungsbeauftragter am IUED.

1. DAC $=$ Development Assistance Committee. Dem Entwicklungshilfeausschuss der OECD gehören 21 Länder an.

2. Quelle: OCDE, Coopération pour le développement. Efforts et politiques des membres du Comité d'aide au développement. Rapport 1996, Paris, OCDE, 1997. 
Einsatzmittel: SKH-Personal, finanzielle Beiträge sowie Nahrungsmittel- und Materiallieferungen. Die Einsatzbereiche sind:

口 Prävention: (z.B. Beobachtung der Vulkantätigkeit in Mittelamerika);

口 Rettung: Bergung und Versorgung von Verletzten, Rettung von verschütteten Personen nach einem Erdbeben;

口 Überleben: Trinkwasserversorgung, Verteilung von Nahrung, Montage von Zelten und Unterkünften, medizinische Betreuung;

口 Wiederaufbau: Wiederherstellung der Infrastruktur (Strassen, Brücken, usw.), Wiederaufbau öffentlicher Gebäude (Spitäler, Heime, Schulen) und Wohnhäuser, Gesundheitsprogramme.

\section{Schema Nr. 1: Einsatzarten der humanitären Hilfe}

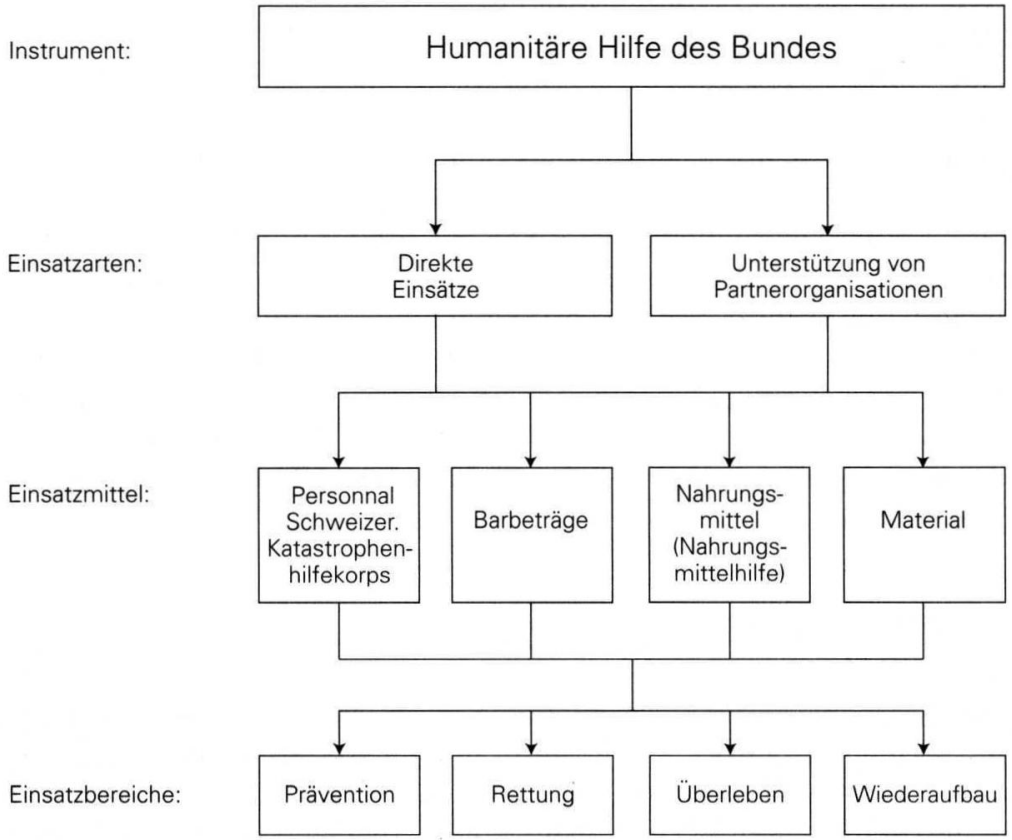

Quelle: Botschaft des Bundesrates über die Weiterführung der internationalen humanitären Hilfe der Eidgenossenschaft, Bern, Botschaft vom 20. November 1996, Nr. 96.092.

In der Öffentlichkeit herrscht oft der Eindruck, dass die humanitäre Hilfe in den letzten Jahren auf Kosten der längerfristigen Entwicklungszusammenarbeit (EZA) sehr stark zugenommen habe. Die Analyse der jüngsten Entwicklung der humanitären Hilfe des Bundes widerlegt diesen Eindruck jedoch, zumindest was die in der öffentlichen EZA enthaltene Hilfe betrifft. Der Anteil der humanitären Hilfe machte seit 1986 zwischen $17,8 \%$ und $22 \%$ der gesamten öffentlichen EZA aus. Seit 1991 ist er sogar rückläufig (Rückgang von 20,4\% 1991 auf $17,8 \% 1997$ der öffentlichen Hilfe). 


\section{Methodische Schwierigkeiten bei der Analyse der humanitären Hilfe}

Die Analyse der neuesten Entwicklung der humanitären Hilfe stösst bei der Auslegung der statistischen Daten auf gewisse Schwierigkeiten: Es gibt kein umfassendes stastistisches Aggregat über humanitäre Hilfe, und die Abgrenzung zwischen längerfristiger Entwicklungszusammenarbeit und Nothilfe wird immer verschwommener.

Mehrere Bereiche der Entwicklungshilfe liegen an der Grenze zwischen humanitärer Hilfe und Entwicklungszusammenarbeit: Konfliktprävention, Hilfe für Strassenkinder, Verteilung von Milch und Nahrung im Rahmen der Unterstützung von Schulen, friedenserhaltende Einsätze der Vereinten Nationen. Am Ende eines Konflikts nimmt die Unterstützung der Wiederaufbauanstrengungen wachsende Bedeutung an. Diese Anstrengungen beschränken sich nicht auf die Instandsetzung der Basisinfrastruktur, den Wiederaufbau von Gebäuden, Spitälern und Schulen, sondern decken einen immer breiteren Bereich $a b$ : Entminungsarbeiten, Rückkehr von Vertriebenen und Flüchtlingen, Wiedereingliederung von demobilisierten Soldaten oder von Rebellen ins Wirtschaftsleben, Förderung des Demokratisierungsprozesses (Überwachung der Wahlen, Stärkung der Justizsysteme, Förderung und Schutz der Menschenrechte, Umstellung der Produktionsanlagen für militärisches Material auf zivile Tätigkeiten).

Aus der Statistik des OECD-Entwicklungshilfeausschusses (DAC) lässt sich der genaue Betrag der humanitären Hilfe nicht ermitteln. Die OECD unterscheidet zwischen der Hilfe für Entwicklungsländer, die in der Statistik der öffentlichen Entwicklungshilfe enthalten ist, und der Hilfe für Transitionsländer, die im Aggregat der öffentlichen Hilfe (mittel- und osteuropäische Länder, wirtschaftlich fortgeschrittenere Entwicklungsländer) eingeschlossen ist. Es ist sehr schwierig, die humanitäre Hilfe der verschiedenen DAC-Länder zu vergleichen, da die vom DAC veröffentlichte Statistik es nicht erlaubt, alle Bestandteile dieser Hilfe in einem getrennten Aggregat humanitärer Hilfe zu erfassen.

In der DAC-Statistik lassen sich folgende Komponenten der humanitären Hilfe aussondern:

- Nahrungsmittelhilfe: Lieferung von Nahrungsmitteln für menschliche Ernährung im Rahmen der Nothilfe, Geldbeiträge zum Kauf von Nahrungsmitteln, Transportkosten der Produkte, Lieferung von Zwischenprodukten im Rahmen der Nahrungsmittelhilfeprogramme (Produkte für tierische Ernährung und landwirtschaftliche Produktionsmittel wie Dünger und Saatgut). Die multilaterale Hilfe in diesem Bereich läuft meistens über das Welternährungsprogramm (WEP). In der DAC-Statistik umfasst die Nahrungsmittelhilfe auch die im Rahmen von Programmen der Entwicklungszusammenarbeit geleistete Nahrungsmittelhilfe.

- Nothilfe bei einer Naturkatastrophe oder einer von Menschen verursachten Katastrophe.

- Die Aufwendungen für die vorübergehende Unterbringung von Flüchtlingen in den Ländern des Nordens können (sofern die Länder dies wünschen) im ersten Aufnahmejahr unter der öffentlichen Entwicklungshilfe verbucht werden. Die meisten Länder, darunter auch die Schweiz, verbuchen diese Ausgaben nicht in ihrer öffentlichen Entwicklungszusammenarbeit. Jedoch machen nach und nach eine zunehmende Anzahl von Ländern von dieser Möglichkeit Gebrauch. Die Aufwendungen für die Wiederansiedlung von Flüchtlingen in einem Hilfeempfängerland können in der öffentlichen EZA aufgeführt werden.

口 Aufwendungen des öffentlichen Sektors für die Unterstützung von Flüchtlingen oder Vertriebenen in den Entwicklungsländern.

Zur vollständigen Untersuchung der humanitären Hilfe müsste man ausserdem folgende Elemente absondern können, was leider nicht der Fall ist:

口 Multilaterale Hilfe: Beiträge an die im humanitären Bereich tätigen multilateralen Organisationen. Es ist nicht möglich, in der DAC-Statistik die für multilaterale Hilfe bestimmten Beträge für die internationalen Organisationen, welche ausschliesslich oder hauptsächlich im humanitären Bereich tätig sind, von den für längerfristige Entwicklungszusammenarbeit bestimmten Beträgen zu trennen. Die Beiträge an das UNHCR und die UNRWA* sind beispielsweise in der Summe der Beiträge für das UN-System (mit allen anderen Organen und operationellen Fonds) enthalten. Zudem arbeiten bestimmte multilaterale Organisationen in beiden Bereichen, ohne dass man diese beiden Aspekte trennen könnte (zum Beispiel Beiträge an die WHO oder die UNICEF). 
a Hilfe für die internationalen privaten Organisationen: Die Beiträge der Schweiz an das IKRK werden unter der bilateralen Hilfe verbucht. Die Beiträge an die internationalen privaten Organisationen umfassen die Beiträge an das IKRK, aber auch an eine Reihe privater Organisationen, die in längerfristigen Projekten tätig sind (zum Beispiel "Entraide universitaire mondiale" oder "Panafrikanisches Entwicklungsinstitut».

Einige mit der humanitären Hilfe zusammenhängende Aspekte werden in der öffentlichen Entwicklungshilfe nicht berücksichtigt:

• Die Aufwendungen der Länder für die Aufnahme von Flüchtlingen in den Ländern des Nordens können nach dem ersten Aufnahmejahr im betreffenden Land nicht als öffentliche Entwicklungshilfe ausgewiesen werden.

• Die Aufwendungen der Länder für die Friedenstruppen werden nicht in der öffentlichen Entwicklungshilfe, sondern in den Militärbudgets aufgeführt.

* UNRWA: Hilfswerk der Vereinten Nationen für die Palästinaflüchtlinge im Nahen Osten.

\section{HAUPTEMPFÄNGERGEBIETE DER HUMANITÄREN HILFE DES BUNDES VON 1990 BIS 1997}

Die Beträge für die humanitäre Hilfe schwanken von einem Jahr zum anderen stärker als die Beträge für die Entwicklungszusammenarbeit, je nach den Katastrophen und neuen Konflikten, die in den verschiedenen Regionen der Erde auftreten. Deshalb hat die Untersuchung der humanitären Hilfe von Jahr zu Jahr nicht viel Sinn, da die Schwankungen sehr stark sein können. Um die Merkmale der humanitären Hilfe der Schweiz besser analysieren zu können, haben wir daher die von 1990 bis 1997 geleistete Hilfe zusammengefasst. Die Tabelle im Anhang I führt die Beträge der humanitären Hilfe des Bundes für humanitäre Einsätze von 1990 bis 1997 auf. Sie hebt die wichtigsten Empfängerländer oder -regionen humanitärer Hilfe (Entwicklungsländer sowie mittel- und osteuropäische Staaten) hervor.

Anhand der Zahlenangaben im Anhang lassen sich mehrere Aspekte hervorheben. Unter den allgemeinen Bemerkungen ist anzuführen, dass die humanitäre Hilfe im grossen und ganzen eher darauf ausgerichtet war, durch menschliche Handlungen hervorgerufene Dramen zu lindern, als Opfern von Naturkatastrophen zu helfen. Interethnische Konflikte und Bürgerkriege haben in den letzten Jahren mehr menschliche Tragödien verursacht als Naturkatastrophen (Überschwemmungen, Zyklone, Dürren, Erdbeben, Vulkanausbrüche, Geländeinstabilitäten). Wenn auch die geleistete Hilfe natürlich vom Auftreten der verschiedenen Katastrophen und Konflikte abhängt, so kann man dennoch feststellen, dass sich unter den Hauptempfängern der schweizerischen humanitären Hilfe einige Schwerpunktländer der längerfristigen Entwicklungszusammenarbeit (Madagaskar, Mosambik, Peru, Tansania) befinden.

Die nachstehende Graphik gibt die Aufteilung der humanitären Hilfe nach Regionen wieder : $43 \%$ der bilateralen Hilfe lassen sich geographisch nicht aufteilen. Dieser Betrag umfasst vor allem die Beiträge der Schweiz für die Einsätze des IKRK. Afrika ist der Kontinent, der von der Schweiz am meisten humanitäre Hilfe erhält. 
Graphik Nr. 1: Aufteilung der bilateralen humanitären Hilfe des Bundes nach Kontinenten (1997, in \% des Gesamtbetrags)

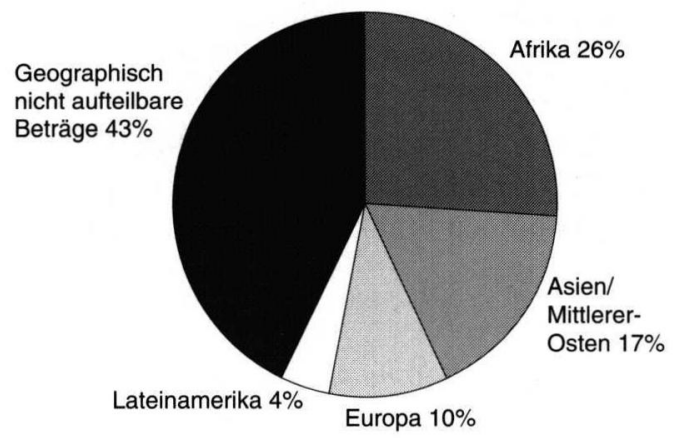

Graphik Nr. 2: Die zehn Hauptempfängerländer und -regionen der humanitären Hilfe des Bundes (Gesamtauszahlungen von 1990 bis 1997, in Millionen Franken)

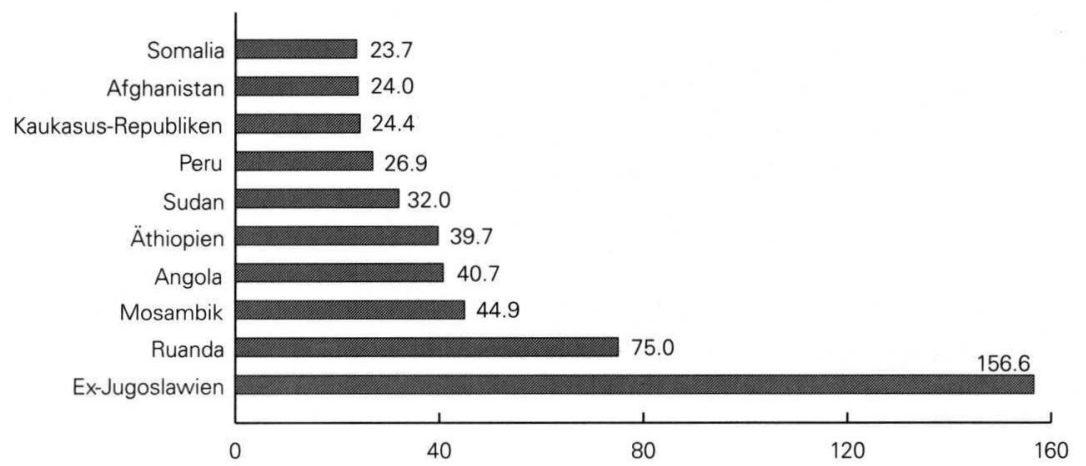

Quellen Graphik 1 und 2: Jahresbericht: Internationale Zusammenarbeit der Schweiz - Humanitäre Hilfe, Bern, DEZA, 1996 und 1997; IUED, Schweizerische Hilfe für Entwicklungsländer und Oststaaten, Genf, IUED, Ausgaben 1991 bis 1997.

In einer genaueren Analyse nach Regionen kann man die wichtigsten von humanitären Katastrophen heimgesuchten Gebiete anführen, in denen die Schweiz einen Grossteil ihrer Hilfe erbracht hat. Die nachstehende Untersuchung bezieht sich auf die Jahre 1990 bis 1997.

\section{$\square$ Europa}

Staaten Ex-Jugoslawiens

Noch nie hatte die Schweiz so viele Mittel in einem Krisengebiet aufgewendet wie in den in Konflikt stehenden Nachfolgestaaten Ex-Jugoslawiens. Die öffentliche humanitäre Entwicklungshilfe des Bundes erreichte von 1991 bis 1997 157 Millionen Franken (sie wurde vor allem zwischen 1992 und 1995 ausgezahlt). Hierzu kommen die in der öffentlichen Entwicklungshilfe nicht berücksichtigten Aspekte: Hilfe für die Flüchtlinge in der Schweiz, ein Teil der vom Bundesamt für Flüchtlinge aufgebrachten Rückkehrhilfe für die bosnischen Flüchtlinge, Aktionen der Schweiz im Rahmen der OSZE, in der Entwicklungszusammenarbeit enthaltene Programme für Wiederaufbauhilfe, Unterstützung 
des Wahlprozesses in Bosnien, Gelbmützeneinsätze, Förderung unabhängiger Medien. Insgesamt beliefen sich die Aufwendungen der Schweiz für diese Staaten von 1991 bis 1996 auf 1,2 Milliarden Dollar. Seit 1996 geht die humanitäre Hilfe zugunsten längerfristiger (in der Entwicklungszusammenarbeit enthaltener) Wiederaufbautätigkeiten zurück.

Russland

Die humanitäre Hilfe für Russland belief sich im Berichtszeitraum auf 14 Millionen Franken (davon 9,4 Millionen Franken 1995 und 1996).

\section{$\square$ Afrika}

Horn von Afrika (Äthiopien, Eritrea, Somalia und Sudan)

Das Horn von Afrika ist mit dem südlichen Afrika das Gebiet des afrikanischen Kontinents, das am meisten Hilfe von der Schweiz erhalten hat. 51,5 Millionen Franken wurden für die Opfer des Konflikts in Äthiopien und das neue Eritrea (vor allem von 1994 bis 1996) ausgezahlt. 55,7 Millionen Franken Hilfe wurden für die Opfer der Dramen in Somalia und im Sudan (hauptsächlich zwischen 1990 und 1993) aufgebracht.

Ruanda/Region der Grossen Seen

Die meisten Programme der Entwicklungszusammenarbeit in diesem Land sind nach den Massakern durcheinandergebracht worden. Die humanitäre Hilfe hat daraufhin sehr grosse Bedeutung gewonnen. Rund 82 Millionen Franken wurden von der Schweiz in Burundi und Ruanda (vor allem seit 1993) aufgewendet.

\section{Mosambik/Malawi}

Für dieses Gebiet wurden 56 Millionen Franken an humanitärer Hilfe ausgezahlt, vor allem um den mosambikanischen Flüchtlingen zu Hilfe zu kommen. Die humanitäre Hilfe für Mosambik erfolgt im Rahmen der Entwicklungszusammenarbeit mit diesem Schwerpunktland der schweizerischen Hilfe.

Angola

41 Millionen Franken an humanitärer Hilfe wurden für die angolanische Bevölkerung aufgebracht.

\section{Nordafrika}

Die humanitäre Hilfe hat sich besonders auf Algerien konzentriert, mit relativ bescheidenen Beträgen (10 Millionen Franken, davon rund die Hälfte 1991). Der Bund leistet vor allem Nahrungsmittelhilfe über die Hilfswerke (davon einen Teil für sahraouische Flüchtlingslager).

Eine ganze Reihe afrikanischer Länder haben Hilfe von der Schweiz erhalten, wobei die Beträge in den meisten Fällen zwischen 10 und 21 Millionen Franken pro Land lagen (nach abnehmender Grössenordnung der Beträge): Madagaskar (Schwerpunktland der Hilfe der Schweiz), mit 21 Millionen Franken, die von 1990 bis 1997 ausgezahlt wurden, die Demokratische Republik Kongo (16 Millionen Franken), Tansania, Liberia, Kenia und Südafrika.

\section{$\square$ Naher und Mittlerer Osten}

In dieser Region hat sich die humanitäre Hilfe auf die israelischen und palästinensischen Gebiete, den Libanon und Jordanien konzentriert. 
In Asien sind folgende Hauptgebiete für den humanitären Einsatz des Bundes zu nennen: Kaukasus-Republiken (Armenien, Aserbeidschan und Georgien, mit Aufwendungen von insgesamt über 24 Millionen Franken, hauptsächlich seit 1994), Kriegsopfer in Afghanistan (24 Millionen Franken), Konflikte und Erdbeben im Iran und Irak (21 Millionen Franken, von 1990 bis 1997 ausgezahlt), Indien und Bangladesch (über 19 Millionen Franken wurden für diese Region aufgebracht), Sri Lanka im Bürgerkrieg (17 Millionen Franken), Kambodscha (14 Millionen Franken). Die Schweiz lässt Nordkorea seit 1995 umfangreiche Nahrungsmittelhilfe zukommen (von 1995 bis 199713 Millionen Franken).

\section{Lateinamerika}

Die für diese Region bestimmte Hilfe war relativ gering. Sie betrifft erstaunlicherweise vor allem Länder, mit denen der Bund und die Nichtregierungsorganisationen (NRO) auch Programme langfristiger Entwicklungszusammenarbeit unterhalten (bedeutende Hilfe für Peru, Brasilien und Chile).

\section{Hilfe für Mittelamerika}

Die humanitäre Hilfe für dieses Schwerpunktgebiet der schweizerischen Entwicklungszusammenarbeit wurde vor allem im Anschluss an die Konflikte in dieser Region aufgebracht (Bürgerkrieg in El Salvador, Konflikte in Nicaragua), insbesondere für die Hilfe für die Vertriebenen und Flüchtlinge in der Region. Sie betrifft aber auch Naturkatastrophen (z.B. Erdbeben in Nicaragua 1992).

\section{Haiti}

Rund 10 Millionen Franken wurden in diesem Land aufgewendet.

\section{VON DEN HILFSWERKEN VERWALTETE HUMANITÄRE HILFE}

Die Spenden der nichtstaatlichen Organisationen für humanitäre Hilfe (private Spenden genannt) werden in der DAC-Statistik berücksichtigt, wenn sie von einer NRO des Nordens an eine NRO des Südens oder an die Empfängerbevölkerung ausgezahlt werden (Geld- oder Sachspenden). Hingegen werden unter «privaten Spenden» alle anderen Zahlungen privaten Ursprungs nicht berücksichtigt, die unter den Begriff «private Transferzahlungen» im Sinne der Zahlungsbilanz fallen (zum Beispiel Geldüberweisungen von Gastarbeitern und Flüchtlingen in Europa an ihre Familien).

Viele private Organisationen sind im Bereich der humanitären Hilfe tätig. Die Statistiken über die humanitäre Hilfe der NRO sind mit Vorsicht zu analysieren, da die NRO bei der Beantwortung jährlicher Erhebungen selbst bestimmen, was ihrer Meinung nach eher unter humanitäre Hilfe oder unter Entwicklungszusammenarbeit fällt. Wegen der recht verschwommenen Abgrenzung zwischen diesen beiden Bereichen kann der Begriff der humanitären Hilfe von der Leitung einer NRO zur anderen unterschiedlich sein. 


\section{$\square$ Gesamtbetrag der von den NRO verwalteten humanitären Hilfe}

Die Finanzierung der humanitären Einsätze der NRO erfolgt durch die öffentlichen Sammlungen der Hilfswerke, durch grosse nationale Sammlungen von Organisationen wie der Glückskette sowie durch die Kantone und Gemeinden. 1996 beliefen sich die Gesamtaufwendungen der NRO für humanitäre Aktionen in den Ländern des Südens und Ostens auf über 95,2 Millionen Franken. 62 Millionen Franken wurden durch Sammlungen in der Bevölkerung und 30 Millionen Franken durch Beiträge des Bundes finanziert. Hinzu kommen 3 Millionen Franken humanitärer Hilfe der Schweizer Kantone und Gemeinden, die über die NRO lief. Die statistischen Angaben zur Finanzierung der Aktionen schweizerischer NRO aus dem Ausland sind sehr lückenhaft (Finanzierung durch Schwester- oder Mutterorganisationen im Ausland, durch die Europäische Union oder durch ausländische Staaten).

Tabelle Nr. 1: Die wichtigsten im Bereich der humanitären Hilfe tätigen schweizerischen privaten Organisationen

\begin{tabular}{lrc}
\hline Wichtigste in der humanitären & \multicolumn{2}{c}{ In tausend Franken } \\
\cline { 2 - 3 } Hilfe tätige NRO & Private Mittel 1997 & Bundesbeiträge 1997 \\
\hline Caritas Schweiz & 15046 & 7904 \\
\hline Terre des hommes Lausanne & 9116 & 4010 \\
\hline Schweizerisches Rotes Kreuz & 7888 & 6804 \\
\hline HEKS & 3223 & 1503 \\
\hline Seraphisches Liebeswerk & 2543 & \\
\hline Mediswiss & 1933 & 20 \\
\hline Kroatisches humanitäres Forum & 1679 & \\
\hline Basel hilft & 1603 & \\
\hline Christian Solidarity International & 1381 & \\
\hline St. Petrus-Claver-Solidarität & 1108 & \\
\hline Stiftung Novartis & 860 & 1200 \\
\hline Heilige Familie Werthenstein & 810 & 202 \\
\hline Amis de Soeur Emmanuelle & 744 & \\
\hline ADRA, Adventisten & 678 & 1100 \\
\hline Médecins sans frontières & 518 & 1607 \\
\hline SAH & 517 & 1114 \\
\hline Mariannhiller Missionare & 509 & 397 \\
\hline Medair & 312 & 574 \\
\hline Heilsarmee & 130 & 638 \\
\hline Terre des hommes Schweiz - Genf & 81 & 625 \\
\hline Basler Mission & 43 & 800 \\
\hline Comité de soutien au peuple sahraoui & 36 & $\mathbf{3 2 2 6 0}$ \\
\hline Solidarité Tiers Monde & 0 & \\
\hline Enfants du Monde & 0 & \\
\hline Agha-Khan-Stiftung & 0 & \\
\hline Obige NRO insgesamt & 51070 & \\
\hline Andere Organisationen & $\mathbf{5 7 0 2}$ & \\
\hline Gesamte Hilfe der NRO & & \\
\hline
\end{tabular}

Quelle: IUED, Schweizerische Hilfe für Entwicklungsländer und Oststaaten, 1997/98, Genf, IUED, 1999. 


\section{$\square$ Über die NRO geleitete humanitäre Hilfe des Bundes}

Ein Teil der humanitären Hilfe des Bundes läuft über die NRO, das heisst sie wird von den privaten Organisationen verwaltet, mit der Finanzierung des Bundes oder mit Lebensmittellieferungen durch den Bund (z.B. Milchpulver). 14 bis $16 \%$ der humanitären Hilfe der Eidgenossenschaft werden auf diese Weise über die NRO geleitet. Die humanitäre Hilfe des Bundes über die NRO belief sich 1997 auf 32,3 Millionen Franken. Sie wurde vor allem über folgende Organisationen geleitet: Caritas Schweiz (1997: 7,9 Millionen Franken), Schweizerisches Rotes Kreuz (6,8 Millionen Franken), Terre des hommes Lausanne (4 Millionen Franken), Heilsarmee, Hilfswerk der evangelischen Kirchen der Schweiz, Médecins sans frontières und Terre des hommes Schweiz - Genf (vgl. zweite Tabellenspalte).

\section{$\square$ Verwendung der Sammelerträge der privaten Organisationen (Eigenmittel)}

Dank der öffentlichen Sammlungen konnten die NRO 1997 humanitäre Einsätze in den Entwicklungsländern in Höhe von 52 Millionen Franken und in den mittel- und osteuropäischen Ländern in Höhe von 6 Millionen Franken finanzieren. Die Tabelle listet die wichtigsten im Bereich der humanitären Hilfe tätigen NRO auf, wobei in der ersten Spalte (Private Mittel) die durch öffentliche Sammlungen finanzierten Aktionen und in der zweiten Spalte die mit Bundesbeiträgen finanzierten Aktionen aufgeführt sind.

\section{$\square$ Die Rolle der Glückskette}

In der Tabelle sind einige Organisationen nicht aufgeführt, die bei den Sammlungen in der Öffentlichkeit eine wichtige Rolle spielen, aber selbst keine humanitären Einsätze verwalten. Zum Beispiel konnten 1996 durch die Sammlungen der Glückskette rund 10,2 Millionen Franken aufgebracht werden. Diese Gelder werden an Hilfswerke überwiesen, um die Aktionen dieser Organisationen zu finanzieren. 1997 hat die Glückskette auf diese Weise 8,5 Millionen Franken an die Hilfswerke für Einsätze in den Entwicklungsländern und 1,25 Millionen Franken für Einsätze in den mittel- und osteuropäischen Ländern ausgezahlt. Die Hilfswerke, die 1997 die höchsten Beiträge über die Glückskette erhielten, sind: Caritas Schweiz (4,8 Millionen Franken), Schweizerisches Rotes Kreuz (3,5 Millionen Franken), Hilfswerk der evangelischen Kirchen der Schweiz (2,1 Millionen Franken) und Terre des hommes Lausanne (1,1 Millionen Franken). $\mathrm{Zu}$ erwähnen ist, dass ein Teil der von der Glückskette nach Katastrophen aufgebrachten Gelder nicht für die klassische Nothilfe verwendet wird, sondern dem Wiederaufbau und der längerfristigen Hilfe in den betroffenen Gebieten dient. Die durch Sammlungen aufgebrachten Gelder können somit auf Aktionen verteilt werden, die sich über mehrere Jahre hinziehen.

\section{$\square$ Hauptempfängerländer der Hilfe der NRO}

Die nachstehenden Graphiken führen die wichtigsten Regionen auf, denen der humanitäre Einsatz der schweizerischen privaten Organisationen in den Jahren 1990 bis 1997 zugute kam. Anhang II gibt die humanitäre Hilfe der NRO für die Hauptempfängerländer an, nach den einzelnen Jahren aufgeteilt. Der Anteil der von den NRO für die osteuropäischen und asiatischen Länder aufgebrachten humanitären Hilfe ist grösser als der Anteil der humanitären Hilfe des Bundes. 
Einige Hauptempfängerländer der Hilfe der NRO stehen auch auf der Liste der Hauptempfängerländer der Hilfe des Bundes (Ex-Jugoslawien, Ruanda, Somalia und Sudan, Armenien). Die Hilfe der NRO für die Nachfolgestaaten des früheren Jugoslawiens, insgesamt 89 Millionen Franken, kommt zur Hilfe des Bundes in Höhe von 157 Millionen Franken hinzu, wobei die privaten Zahlungen der starken bosnischen Bevölkerung in der Schweiz nicht berücksichtigt sind (Geschenke für ihre Familien und nahen Verwandten und für bestimmte Organisationen, die die Fragebögen über Zahlungen im Ausland nicht ausfüllen). Die Hilfe für den Irak betrifft vor allem die humanitäre Hilfe für die Kurden (insbesondere durch die von der Glückskette gesammelten umfangreichen Spendengelder).

\section{Graphik Nr. 3: Aufteilung der humanitären Hilfer der NRO nach Kontinenten} (1997, in \% des Gesamtbetrags)

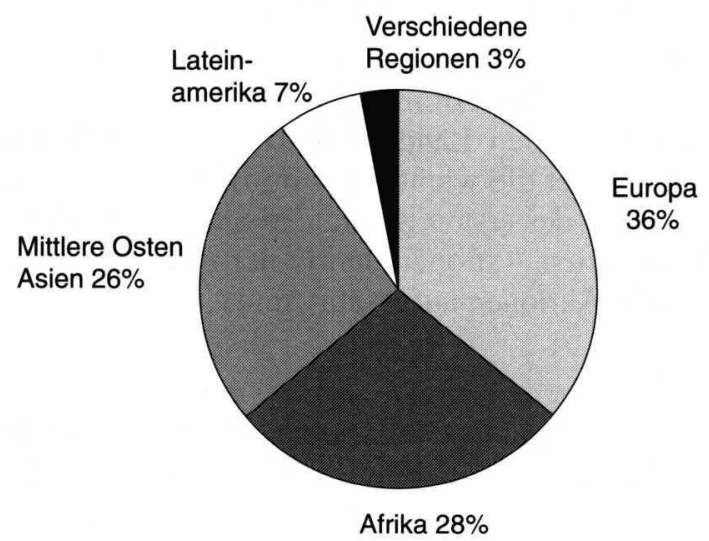

Graphik Nr. 4: Die zehn Hauptempfängerländer und -regionen

der humanitären Hilfe der NRO (aus Eigenmitteln finanzierte Aktionen)

(Gesamtaufwendungen von 1990 bis 1997, in Millionen Franken)

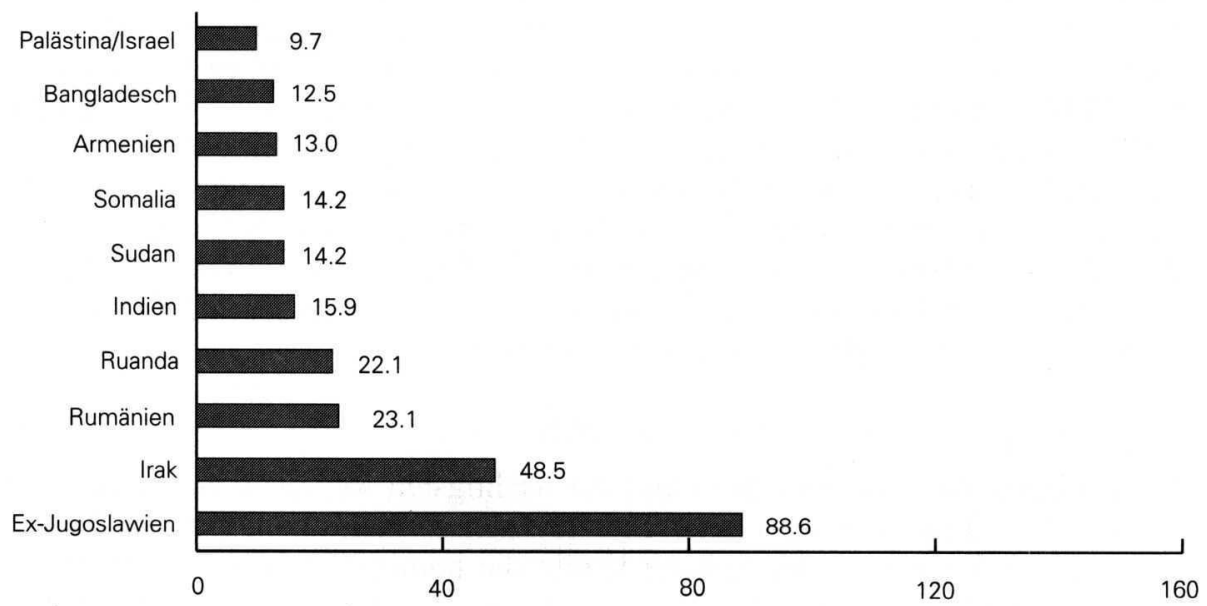

Quelle Graphik 3 und 4: Berechnung anhand der statistischen Angaben der IUED-Publikation "Schweizerische Hilfe für Entwicklungsländer und Oststaaten », Jahrgänge 1991 bis 1997/98. 


\section{Rolle der öffentlichen Sammlungen}

Die Rolle der privaten Organisationen ist bei der Mobilisierung der öffentlichen Meinung und beim Spendensammeln sehr wichtig. Ein Grossteil der bei verschiedenen Sammlungen in der Öffentlichkeit aufgebrachten Gelder hängt auf die eine oder andere Weise mit der Reaktion der Medien auf bestimmte Katastrophen zusammen. Die Beziehungen zwischen den NRO und den Medien sind zwiespältig, ja inzestuös. Die NRO brauchen eine Reaktion der Medien auf gewisse Katastrophen, um anschliessend Sammelkampagnen lancieren zu können. Man kann sich manchmal fragen, ob nicht die schlimmsten Katastrophenmeldungen am meisten Spendeneingänge einbringen. Die Medien schenken im allgemeinen Notsituationen mehr Beachtung als Entwicklungssituationen, weil die spektakuläre Seite beim Fernsehzuschauer mehr Emotionen und eine gespanntere Aufmerksamkeit erregt. Das wiederholte Auftreten von durch Menschen verursachten humanitären Katastrophen und die Konflikte können indes langfristig zu einer gewissen Spendenmüdigkeit führen. Ein Artikel in der Zeitschrift Mosquito von 1997 trug den provozierenden Titel «Unsere tägliche Katastrophe gib uns heute... $»^{3}$.

Gewisse Themen sind ergiebiger als andere, wenn man Geld in der Öffentlichkeit sammeln will. Geld sammeln, um Hungernden oder Strassenkindern zu helfen, ist zweifellos einfacher als Geld zu sammeln, um den Flüchtlingen (hier oder anderswo) zu helfen oder die Aktion von Gewerkschaften in der Dritten Welt zu unterstützen. Es genügt, die massenweise Verwendung von Photos mit notleidenden Kindern in den Werbesendungen und Zeitungen von Organisationen zu sehen, um zu begreifen, dass dies sicher beim Spendensammeln einträglicher ist als das Bild eines farbigen Mannes. Ein weiterer Motivationsfaktor für den Spender und die Spenderin ist, dass rund die Hälfte der von den Hilfswerken in der Schweiz aufgebrachten Gelder durch Missionsorganisationen oder den Kirchen nahestehende Organisationen gesammelt wird. Selbst wenn die Missionen natürlich nicht nur in der Nothilfe arbeiten, sondern auch längerfristige Entwicklungsprojekte unterstützen, lässt uns diese Tatsache dennoch einen Teil der Motivationen der Spender und Spenderinnen erkennen (Bedeutung der Spende aus christlicher Nächstenliebe).

\section{Vielzahl von am gleichen Ort arbeitenden humanitären Akteuren}

Hunderte von schweizerischen Hilfswerken (oder schweizerischen Zweigstellen internationaler Organisationen) sind im humanitären Bereich tätig, was mehr als einen Spender und eine Spenderin verwirren muss, die mit der Vielzahl der Werbesendungen im Briefkasten konfrontiert werden. Auch kann man sich die Koordinationsprobleme vor Ort vorstellen. In einem Gebiet wie Bosnien steht das Empfängerland fünfzehn internationalen Organisationen, Dutzenden von Regierungsstellen und Hunderten von NRO gegenüber. Im Fall der Schweiz allein trifft man vor Ort Vertreter und Vertreterinnen des EDA, der DEZA (und des Schweizerischen Katastrophenhilfekorps), des Bundesamtes für Flüchtlinge, des Departements für Verteidigung, Bevölkerungsschutz und Sport (VBS) und von fünfzehn schweizerischen NRO an. Es bleibt noch enorm viel Arbeit, um einen besseren Informationsfluss zu erreichen und die Koordination unter den NRO zu verbessern.

\footnotetext{
3. Mosquito Nr. 4, Juni 1997.
} 


\section{QUELLEN}

Zur Statistik der humanitären Hilfe sowie zu den Aktionen des Bundes und der schweizerischen NRO :

OCDE, Coopération pour le développement. Efforts et politiques des membres du Comité d'aide au développement. Rapport 1996, Paris, OCDE, 1997.

OCDE, Coopération pour le développement. Efforts et politiques des membres du Comité d'aide au développement. Rapport 1997, Paris, OCDE, 1998.

Botschaft des Bundesrates über die Weiterführung der internationalen humanitären Hilfe der Eidgenossenschaft, vom 20. November 1996, Bern, Nr. 96.092.

DEZA, Jahresbericht Internationale Zusammenarbeit der Schweiz, Humanitäre Hilfe 1997, Bern, 1998.

DDC, Corps suisse d'aide en cas de catastrophe (ASC), brochure de présentation, 1998, 64 S.

IUED/DEZA, Schweizerische Hilfe für Entwicklungsländer und Oststaaten 1996/1997, Genf, IUED, 1998.

(Die Broschüre enthält ein Verzeichnis der in der Entwicklungszusammenarbeit und der humanitären Hilfe tätigen NRO, die Beträge der von den NRO aufgebrachten Leistungen sowie die bilaterale Hilfe des Bundes nach Empfänger ländern aufgeteilt).

Mosquito, Die entwickungspolitische Zeitschrift der Schweiz, Nr. 4, Juni 1997 (Dossier: Spendenmarkt Schweiz: Umfang, Strategien, Gewinner, Verlierer).

IUED, Etude sur l'évolution de l'aide humanitaire des pays membres du CAD, Genf, 1993 (unveröffentlichter Bericht für das IKRK).

Zur humanitären Hilfe im allgemeinen :

(Unter der Leitung von) Claire Pirotte und Bernard Husson, Entre urgence et développement. Pratiques humanitaires en question, Paris, Ed. Karthala, 1997, 238 S.

Myriam Donsimoni, Du don à l'aide. Le marché de l'altruisme, Paris, L'Harmattan, 1995, $228 \mathrm{~S}$.

René Holenstein, Was kümmert uns die Dritte Welt. Zur Geschichte der internationalen Solidarität in der Schweiz, Zürich, Chronos Verlag, 1998, 264 S.

(Unter der Leitung von) Myriam Tsikounas (Gemeinschaftswerk), Les ambiguïtés de l'humanitaire, Editions Corlet, 1996, (Vertrieb Le Seuil), 190 S.

Gemeinschaftswerk, ONG et développement. Société, économie, politique. Paris, Ed. Karthala, 1998, 684 S.

Coopération internationale : le temps des incertitudes, Dossier in: Revue Tiers Monde, Nr. 151, Juli-September 1997.

INTERNET-ADRESSEN

DEZA, Direktion für Entwicklung und Zusammenarbeit : http//www.sdc-gov.ch

Schweizerisches Katastrophenhilfekorps : http//www.skh.ch 
Anhang I: Entwicklung der humanitären Hilfe des Bundes für die Hauptempfängerländer (öffentliche Entwicklungshilfe und öffentliche Hilfe) in Mio. Franken

\begin{tabular}{|c|c|c|c|c|c|c|c|c|c|}
\hline $\begin{array}{l}\text { Land// } \\
\text { Region }\end{array}$ & 1990 & 1991 & 1992 & 1993 & 1994 & 1995 & 1996 & $1997^{\mathrm{C}}$ & $\begin{array}{l}\text { esamt- } \\
\text { betrag }\end{array}$ \\
\hline \multicolumn{10}{|l|}{ Europa } \\
\hline Ex-Jugoslawien & 0.00 & 2.62 & 26.00 & 39.64 & 27.54 & 30.68 & 16.90 & 13.21 & 156.6 \\
\hline Russland & & & & 0.46 & 2.29 & 5.32 & 4.06 & 1.67 & 13.8 \\
\hline Albanien & & 0.73 & 2.08 & 0.59 & 0.22 & 0.54 & 0.04 & 0.44 & 4.6 \\
\hline \multicolumn{10}{|l|}{ Afrika } \\
\hline Ruanda & 0.34 & 0.96 & 5.55 & 10.19 & 20.18 & 16.92 & 13.37 & 7.51 & 75.0 \\
\hline Mosambik & 5.74 & 5.57 & 13.39 & 6.94 & 6.53 & 4.40 & 1.92 & 0.44 & 44.9 \\
\hline Angola & 5.62 & 2.43 & 1.92 & 3.96 & 4.88 & 4.18 & 9.41 & 8.30 & 40.7 \\
\hline Äthiopien & 4.07 & 12.78 & 3.53 & 3.92 & 2.17 & 5.31 & 5.58 & 2.30 & 39.7 \\
\hline Sudan & 5.77 & 4.84 & 4.88 & & 2.33 & 4.32 & 3.25 & 3.03 & 32.0 \\
\hline Somalia & 2.45 & 4.98 & 5.06 & & 0.79 & 2.34 & 1.55 & 3.84 & 23.7 \\
\hline Madagaskar & 0.85 & 1.53 & 3.06 & 1 & .51 & 2.98 & 1.70 & 1.77 & 21.3 \\
\hline Kongo lex-Zai & 0.63 & 2.29 & 0.60 & 7 & 3.22 & 1.17 & 4.77 & 2.29 & 16.1 \\
\hline Tansania & 1.36 & 0.86 & 1.88 & 0.93 & 5.62 & 0.68 & 1.05 & 2.12 & 14.5 \\
\hline Liberia & 0.20 & 0.50 & 2.04 & 0.28 & 2.53 & 3.53 & 2.40 & 1.62 & 13.1 \\
\hline Eritrea & & & & 0.00 & 4.23 & 4.39 & 1.61 & 1.56 & 11.8 \\
\hline Malawi & 2.85 & 3.63 & 2.58 & 0.27 & 1.46 & 0.40 & 0.24 & 0.11 & 11.5 \\
\hline Kenia & 0.05 & 1.78 & 2.57 & 1.72 & 1.69 & 1.46 & 0.78 & 0.97 & 11.0 \\
\hline Kap Verden & 0.57 & 0.86 & 1.26 & 0.81 & 1.58 & 1.66 & 2.03 & 1.52 & 10.3 \\
\hline Algerien & 0.69 & 4.77 & 0.83 & & 0.86 & 0.73 & 0.67 & 0.57 & 10.2 \\
\hline Südafrika & 1.32 & 1. & 7 & 1 & 3 & 0.53 & 0.39 & 0.37 & 10.1 \\
\hline Uganda & 2. & 2 & 2 & 7 & 0.20 & 0.13 & & & 1.1 \\
\hline Simb & 0.2 & 0 & 30 & 1 & 0.54 & 0.63 & 0.18 & 0.33 & .5 \\
\hline Burundi & 0.1 & 0 & 0 & 0 & 1.34 & 0.53 & 2.14 & 1.24 & 6.9 \\
\hline Burkina Faso & 0.50 & 1.46 & 1.30 & 0.79 & 0.56 & 0.68 & 1.03 & 0.58 & 6.9 \\
\hline Sambia & 0.4 & 0.83 & 1.73 & 0.62 & 0.58 & 1.03 & 0.39 & 0.38 & 6.1 \\
\hline Ischad & 0.2 & 1.98 & 0.2 & 0 & 0.71 & 0.30 & 0.1 & 0.30 & 4.8 \\
\hline Senegal & 1.29 & 0 & 0.60 & 0.44 & 0.57 & 0.38 & 0.34 & 0.34 & 4.8 \\
\hline Mauretanien & 0.28 & 0.45 & 0.87 & 1.32 & 0.17 & 0.26 & 0.71 & 0.20 & 4.3 \\
\hline Mali & 0.05 & 0.85 & 0.26 & 0.02 & 0.13 & 0.10 & 0.94 & 1.59 & 3.9 \\
\hline Afrika insgesam & & & & & 71.80 & 61.69 & 58.68 & 46.81 & \\
\hline \multicolumn{10}{|l|}{ Asien/Mittle } \\
\hline \multicolumn{4}{|c|}{ Kaukasus-Republiken } & 1.02 & 5.70 & 5.02 & 4.44 & 8.20 & 24.4 \\
\hline Afghanistan & 2.70 & 3.61 & 5.41 & 2.00 & 2.00 & 3.80 & 1.80 & 2.70 & 24.0 \\
\hline Sri Lanka & 2.33 & 2.33 & & & 2.40 & 1.63 & 1.37 & 1.03 & 16.6 \\
\hline Icrael & 2.01 & 383 & 010 & 20 & 1.61 & 0.50 & 0.03 & 0.50 & 14.0 \\
\hline Kambod & 0.86 & 3.91 & 1.96 & 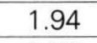 & 2.79 & 1.51 & 0.41 & 0.34 & 13.7 \\
\hline Nordkorea & & & & 0.00 & 0.00 & 4.33 & 2.79 & 6.32 & 13.4 \\
\hline Libanon & 2.40 & 2.56 & 1.36 & 1.17 & 1.40 & 0.72 & 1.54 & 1.10 & 12.3 \\
\hline Irak & & 1.79 & 2.34 & 0.06 & 0.11 & 1.11 & 2.81 & 2.54 & 10.8 \\
\hline Iran & 3.15 & 5.92 & 0.01 & 0.55 & 0.05 & & 0.04 & 0.52 & 10.2 \\
\hline Indien & 2.58 & 2.00 & 1.93 & 1.38 & 0.49 & 0.80 & 0.27 & 0.47 & 9.9 \\
\hline Jordanien & 0.68 & 8.37 & 0.00 & 0.00 & 0.00 & 0.50 & & & 9.5 \\
\hline Bangladesch & 0.93 & 2.57 & 3.14 & 0.53 & 0.76 & 0.58 & 0.41 & 0.27 & 9.2 \\
\hline Thailand & 3.48 & 2.51 & 0.59 & 0.11 & 1.17 & 0.26 & 0.25 & 0.50 & 8.9 \\
\hline Palästina & & & & 3.00 & 0.69 & 1.76 & 1.39 & 0.78 & 7.6 \\
\hline Vietnam & 0.78 & 0.16 & 1.20 & 1.14 & 1.74 & 1.08 & 0.35 & 0.82 & 7.3 \\
\hline Tadschikistan & & & & 0.50 & 1.30 & 0.86 & 1.82 & 2.12 & 6.6 \\
\hline Philippinen & 1.44 & 1.47 & 1.33 & 0.53 & 0.43 & 0.16 & 0.14 & 0.27 & 5.8 \\
\hline Nepal & 0.53 & 0.23 & 0.39 & 0.86 & 1.37 & 0.70 & 0.22 & 0.15 & 4.4 \\
\hline
\end{tabular}




\begin{tabular}{|c|c|c|c|c|c|c|c|c|c|}
\hline $\begin{array}{l}\text { Land/ } \\
\text { Region }\end{array}$ & 1990 & 1991 & 1992 & 1993 & 1994 & 1995 & 1996 & $1997^{\mathrm{G}}$ & $\begin{array}{r}\text { Gesamt- } \\
\text { betrag }\end{array}$ \\
\hline Indonesien & 0.26 & 0.00 & 0.03 & 0.32 & 0.61 & 0.35 & 0.19 & 0.68 & 2.4 \\
\hline China & 0.16 & 0.30 & 0.30 & 0.05 & 0.01 & 0.24 & 0.49 & 0.41 & 2.0 \\
\hline Asien insgesam & & & & & 27.29 & 26.26 & 21.63 & 29.92 & \\
\hline \multicolumn{10}{|l|}{ Lateinamerika } \\
\hline Peru & 4.47 & 5.08 & 4.73 & 3.27 & 3.03 & 2.12 & 2.24 & 1.99 & 26.9 \\
\hline Brasilien & 2.05 & 1.73 & 2.33 & 2.09 & 2.03 & 2.07 & 1.37 & 1.34 & 15.0 \\
\hline Chile & 1.99 & 1.58 & 1.63 & 1.31 & 1.21 & 1.09 & 0.89 & 0.80 & 10.5 \\
\hline Haiti & 0.90 & 0.78 & 1.76 & 0.79 & 2.18 & 1.44 & 0.96 & 0.85 & 9.6 \\
\hline Kolumbien & 0.47 & 0.22 & 0.28 & 0.22 & 0.70 & 1.15 & 0.74 & 1.08 & 4.9 \\
\hline Guatemala & 0.23 & 0.53 & 1.19 & 0.07 & 0.63 & 0.54 & 0.51 & 0.64 & 4.3 \\
\hline Ekuador & 0.21 & 0.36 & 0.43 & 0.49 & 0.82 & 0.75 & 0.72 & 0.47 & 4.2 \\
\hline Nicaragua & 0.95 & 0.44 & 0.54 & 0.61 & 0.80 & 0.21 & 0.15 & 0.14 & 3.8 \\
\hline \multicolumn{6}{|c|}{ Lateinamerika insgesamt } & 13.16 & 10.17 & 8.00 & 7.86 \\
\hline \multicolumn{6}{|c|}{ Geogr. nicht aufgeteilt } & 65.87 & 68.17 & 73.93 & 74.49 \\
\hline \multirow{2}{*}{\multicolumn{5}{|c|}{$\begin{array}{l}\text { Multilaterale Hilfe } \\
\text { Hilfe insgesamt }\end{array}$}} & 44.68 & 41.22 & 46.46 & 44.60 & \\
\hline & & & & & 253.67 & 244.76 & 230.40 & 222.11 & \\
\hline Davon öff. EZA & 190.40 & 228.70 & 245.20 & 236.10 & 250.70 & 238.80 & 225.70 & 217.10 & \\
\hline $\begin{array}{l}\text { Hilfe in \% } \\
\text { der öff. EZA }\end{array}$ & 19.06 & 20.37 & 18.11 & 19.72 & 19.27 & 19.32 & 17.83 & 17.84 & \\
\hline
\end{tabular}

Anhang II: Entwicklung der humanitären Hilfe der NRO, in Millionen Franken Hauptempfängerländer der humanitären Einsätze der NRO

\begin{tabular}{lrrrrrrrrr}
\hline $\begin{array}{l}\text { Land/ } \\
\text { Region }\end{array}$ & $\mathbf{1 9 9 0}$ & $\mathbf{1 9 9 1}$ & $\mathbf{1 9 9 2}$ & $\mathbf{1 9 9 3}$ & $\mathbf{1 9 9 4}$ & $\mathbf{1 9 9 5}$ & $\mathbf{1 9 9 6}$ & $\mathbf{1 9 9 7} \begin{array}{r}\text { Gesamt- } \\
\text { betrag }\end{array}$ \\
\hline Europa & & & & & & & & & \\
\hline Ex-Jugoslawien & 0.00 & 1.22 & 4.41 & 11.84 & 22.87 & 16.35 & 18.47 & 13.42 & $\mathbf{8 8 . 6}$ \\
\hline Rumänien & 3.40 & 5.63 & 2.43 & 2.79 & 1.80 & 1.34 & 3.00 & 2.72 & $\mathbf{2 3 . 1}$ \\
\hline Türkei & 0.02 & 4.10 & 0.56 & 1.03 & 0.03 & 0.03 & 0.24 & 0.12 & $\mathbf{6 . 1}$ \\
\hline Russland & & 0.08 & 0.26 & 0.05 & 0.31 & 1.87 & 1.29 & 0.59 & $\mathbf{4 . 4}$ \\
\hline Bulgarien & 0.22 & 0.76 & 0.08 & 0.20 & 0.21 & 0.14 & 1.00 & 0.58 & $\mathbf{3 . 2}$ \\
\hline Polen & 0.16 & 0.46 & 0.73 & 0.35 & 0.35 & 0.21 & 0.07 & 0.58 & $\mathbf{2 . 9}$ \\
\hline Albanien & & 0.12 & 0.22 & 0.26 & 0.25 & 0.22 & 0.54 & 1.11 & $\mathbf{2 . 7}$ \\
\hline Südeuropa & & & & & & & & & \\
insgesamt & $\mathbf{3 . 9 8}$ & $\mathbf{5 . 4 5}$ & $\mathbf{1 0 . 8 7}$ & $\mathbf{1 3 . 1 4}$ & $\mathbf{2 3 . 1 6}$ & $\mathbf{1 6 . 6 5}$ & $\mathbf{1 9 . 2 4}$ & $\mathbf{1 4 . 8 1}$ & \\
\hline Osteuropa & & $\mathbf{1 2 . 4 9}$ & $\mathbf{7 . 3 9}$ & $\mathbf{4 . 0 1}$ & $\mathbf{3 . 0 6}$ & $\mathbf{3 . 8 7}$ & $\mathbf{6 . 0 0}$ & $\mathbf{5 . 9 9}$ & \\
\hline Afrika & & & & & & & & & \\
\hline Ruanda & 0.10 & 0.07 & 0.44 & 0.57 & 10.19 & 5.03 & 2.77 & 2.94 & $\mathbf{2 2 . 1}$ \\
\hline Sudan & 0.39 & 1.64 & 1.23 & 1.60 & 2.73 & 1.28 & 2.21 & 3.10 & $\mathbf{1 4 . 2}$ \\
\hline Somalia & 0.00 & 0.03 & 3.55 & 3.95 & 1.71 & 1.55 & 1.64 & 1.76 & $\mathbf{1 4 . 2}$ \\
\hline Mosambik & 0.24 & 2.17 & 1.03 & 1.49 & 1.52 & 0.77 & 0.56 & 0.34 & $\mathbf{8 . 1}$ \\
\hline Athiopien & 1.88 & 2.34 & 0.58 & 1.39 & 0.78 & 0.27 & 0.54 & 0.20 & $\mathbf{8 . 0}$ \\
\hline Madagaskar & 0.58 & 0.48 & 0.92 & 0.63 & 0.70 & 0.64 & 1.10 & 1.09 & $\mathbf{6 . 1}$ \\
\hline Uganda & 0.58 & 0.67 & 1.30 & 1.73 & 0.83 & 0.17 & 0.19 & 0.12 & $\mathbf{5 . 6}$ \\
\hline Tansania & 1.13 & 0.40 & 0.58 & 0.65 & 1.29 & 0.44 & 0.31 & 0.40 & $\mathbf{5 . 2}$ \\
\hline Simbabwe & 0.33 & 0.06 & 2.20 & 0.29 & 0.13 & 1.45 & 0.04 & 0.05 & $\mathbf{4 . 5}$ \\
\hline Burkina Faso & 0.64 & 1.71 & 0.02 & 0.05 & 0.06 & 0.00 & 0.18 & 0.04 & $\mathbf{2 . 7}$ \\
\hline Südafrika & 0.21 & 0.35 & 0.25 & 0.45 & 0.23 & 0.30 & 0.15 & 0.54 & $\mathbf{2 . 5}$ \\
\hline Sambia & 0.33 & 0.16 & 0.36 & 0.22 & 0.59 & 0.25 & 0.37 & 0.17 & $\mathbf{2 . 5}$ \\
\hline Kongo (ex-Zaire) & & 0.08 & 0.41 & 0.46 & 0.24 & 0.14 & 0.07 & 0.86 & $\mathbf{2 . 3}$ \\
\hline & & & & & & & & &
\end{tabular}




\begin{tabular}{|c|c|c|c|c|c|c|c|c|c|}
\hline $\begin{array}{l}\text { Land/ } \\
\text { Region }\end{array}$ & 1990 & 1991 & 1992 & 1993 & 1994 & 1995 & 1996 & $1997^{C}$ & $\begin{array}{r}\text { Gesamt- } \\
\text { betrag } \\
\end{array}$ \\
\hline Kenia & 0.04 & 0.06 & 0.44 & 0.34 & 0.27 & 0.46 & 0.13 & 0.30 & 2.0 \\
\hline Burundi & 0.00 & 0.10 & 0.02 & 0.21 & 0.22 & 0.24 & 0.58 & 0.64 & 2.0 \\
\hline $\begin{array}{l}\text { Hilfe für } \\
\text { die Sahraouis }\end{array}$ & 0.06 & & 0.31 & 0.20 & 0.46 & 0.45 & 0.29 & 0.12 & 1.9 \\
\hline Eritrea & 0.74 & 0.00 & 0.11 & 0.22 & 0.18 & 0.11 & 0.01 & 0.14 & 1.5 \\
\hline Afrika insgesamt & 9.57 & 13.39 & 16.58 & 18.04 & 23.79 & 16.31 & 14.85 & 15.96 & \\
\hline \multicolumn{10}{|c|}{ Asien/Mittlerer Osten } \\
\hline Irak & 0.00 & 13.04 & 19.78 & 10.81 & 0.51 & 0.77 & 2.22 & 1.35 & 48.5 \\
\hline Indien & 1.87 & 1.57 & 2.11 & 1.94 & 1.22 & 1.43 & 3.21 & 2.61 & 15.9 \\
\hline Armenien & 0.16 & 5.24 & 3.54 & 1.06 & 0.68 & 1.52 & 0.45 & 0.36 & 13.0 \\
\hline Bangladesch & 0.37 & 3.98 & 0.65 & 2.12 & 0.34 & 0.31 & 2.09 & 2.62 & 12.5 \\
\hline Palästina/Israel & 0.00 & 3.04 & 5.09 & 0.05 & 0.09 & 0.02 & 0.69 & 0.74 & 9.7 \\
\hline Philippinen & 0.58 & 2.06 & 0.55 & 0.44 & 0.06 & 0.58 & 2.52 & 2.51 & 9.3 \\
\hline Libanon & 0.96 & 1.14 & 1.15 & 0.89 & 0.12 & 0.20 & 1.21 & 1.17 & 6.8 \\
\hline Iran & 1.15 & 2.09 & 0.39 & 0.54 & 0.39 & & & 0.40 & 5.0 \\
\hline Asien insgesamt & 5.88 & 28.31 & 31.84 & 20.88 & 5.26 & 7.58 & 16.39 & 15.10 & \\
\hline \multicolumn{10}{|l|}{ Lateinamerika } \\
\hline Brasilien & 0.34 & 0.91 & 0.55 & 0.39 & 0.45 & 0.48 & 1.52 & 0.55 & 5.2 \\
\hline Kolumbien & 0.34 & 0.19 & 0.44 & 0.27 & 0.27 & 0.73 & 0.41 & 0.61 & 3.2 \\
\hline Peru & 0.26 & 0.25 & 0.51 & 0.11 & 0.15 & 0.19 & 0.78 & 0.85 & 3.1 \\
\hline Chile & 0.60 & 0.21 & 0.20 & 0.25 & 0.30 & 0.26 & 0.69 & 0.35 & 2.9 \\
\hline El Salvador & 1.16 & 0.41 & 0.58 & 0.35 & 0.23 & 0.03 & 0.02 & 0.00 & 2.8 \\
\hline Nicaragua & 0.73 & 0.32 & 0.40 & 0.47 & 0.20 & 0.17 & 0.14 & 0.05 & 2.5 \\
\hline Haiti & 0.08 & 0.08 & 0.12 & 0.24 & 0.17 & 0.42 & 0.49 & 0.43 & 2.0 \\
\hline Bolivien & 0.06 & 0.07 & 0.11 & 0.34 & 0.10 & 0.10 & 0.18 & 0.70 & 1.7 \\
\hline Guatemala & 0.49 & 0.02 & & 0.05 & 0.17 & 0.22 & 0.06 & 0.40 & 1.4 \\
\hline $\begin{array}{l}\text { Lateinamerika } \\
\text { insgesamt }\end{array}$ & 4.77 & 2.99 & 3.42 & 3.17 & 2.39 & 2.75 & 4.61 & 4.26 & \\
\hline $\begin{array}{l}\text { Geog. } \\
\text { nicht aufgeteilt }\end{array}$ & 3.28 & 0.14 & 0.10 & & 0.27 & 3.73 & 0.56 & 1.69 & \\
\hline $\begin{array}{l}\text { Hilfe im Süden } \\
\text { insgesamt }\end{array}$ & 27.48 & 50.41 & 62.83 & 55.32 & 54.91 & 47.04 & 55.66 & 51.82 & \\
\hline Hilfe im Osten ins & sg. & 12.49 & 7.39 & 4.01 & 3.06 & 3.87 & 6.00 & 5.99 & \\
\hline $\begin{array}{l}\text { Beiträge der DEZA } \\
\text { für die NRO }\end{array}$ & 30.57 & 28.05 & 42.44 & 39.90 & 40.40 & 38.10 & 30.50 & 32.26 & \\
\hline $\begin{array}{l}\text { Hilfe der NRO im s } \\
\text { (Eigenmittel) }\end{array}$ & $\begin{array}{l}\text { Süden } \\
27.48\end{array}$ & 50.41 & 62.83 & 55.32 & 54.91 & 47.04 & 55.66 & 51.82 & \\
\hline $\begin{array}{l}\text { Hilfe der NRO } \\
\text { im Osten }\end{array}$ & & 12.49 & 7.39 & 4.01 & 3.06 & 3.87 & 6.00 & 60.00 & \\
\hline NRO insgesamt & & 90.95 & 112.65 & 99.23 & 98.37 & 89.01 & 92.16 & 90.08 & \\
\hline
\end{tabular}

Quellen: DEZA, Jahresbericht: Internationale Zusammenarbeit der Schweiz - Humanitäre Hilfe, DEZA, Bern, Jahrgänge1990 bis 1997; IUED/DDC, Schweizerische Hilfe für Entwicklungsländer und Oststaaten, IUED, Genf, Jahrgänge 1990 bis 1997. 